\title{
CUATRO NUEVOS REGISTROS DE ORCHIDACEAE PARA HONDURAS
}

\author{
Dora Elisa Pérez Munguía
}

\author{
Universidad Nacional Autónoma de Honduras, Tegucigalpa, Honduras \\ doraelisa.perez53@gmail.com
}

\begin{abstract}
Four new records for the Honduran orchid flora are recorded: Chysis bruennowiana (department of Comayagua), Lycaste cruenta var. minor (Ocotepeque), Ornithidium tonsoniae (Comayagua) and Sobralia powellii (Ocotepeque).

Resumen. Se reportan cuatro nuevos registros para la orquideoflora de Honduras: Chysis bruennowiana (departamento de Comayagua), Lycaste cruenta var. minor (Ocotepeque), Ornithidium tonsoniae (Comayagua) y Sobralia powellii (Ocotepeque).
\end{abstract}

Key words / Palabras Clave: Comayagua, Ocotepeque, orquídeas

Introducción. Honduras es un país con una extensión de $112,492 \mathrm{~km}^{2}$ y está ubicado en el centro de América Central. En su compleja geografía, sobresalen tres regiones: las llanuras de la costa del Caribe, la región montañosa compuesta por tres cordilleras (norte, central y del sur) y las llanuras del golfo de Fonseca. El estudio se realizó en dos zonas: en la Sierra de Comayagua, de la cordillera central y en la Sierra del Merendón, en el departamento de Ocotepeque, que pertenece a la cordillera del sur, atraviesa Honduras desde este departamento en el occidente, hasta el departamento de Gracias a Dios, en el este del país. Las tres regiones están divididas por valles de profundos ríos (Mejía \& House 2002).

La heterogeneidad de hábitats a diferentes elevaciones, así como la espacial, dada por los efectos de montaña y los parteaguas, entre otros, afectan la distribución y abundancia de las poblaciones de orquídeas, incrementando su diversidad (Heywood 1995). El clima de Honduras es tan variado como la configuración de su suelo, y esto también afecta la diversidad y el endemismo.

Aunque los investigadores residentes tengan conocimiento que poblaciones de determinadas especies se encuentran en el país, al no estar éstas documentadas formalmente, la comunidad internacional no tiene acceso a esta información. Esta situación ha hecho que investigadores como Ossenbach, Pupulin y Dressler (2007) digan que Honduras es como un "agujero negro" en cuanto a la información sobre la distribución y abundancia de orquídeas en Centroamérica. El número de registros proporcionado por Nelson Sutherland (2008), quien registró 146 géneros y 652 especies de Orchidaceae, se ha venido incrementando recientemente con los trabajos de investigadores residentes, como los de Linares y Ancalmo (2016), Pérez Munguía (2015a, b), y Vega y colaboradores $(2014,2016)$.

Nuevos Registros para la flora de Honduras

1. Chysis bruennowiana Rchb.f. \& Warsz., Bot. Zeit. (Berlin) 15 (10): 157. 1857. TIPO: Perú. Sin localidad específica, J. Warczewicz s.n. (holotipo, W, no visto).

Hierba epífita de hasta $79 \mathrm{~cm}$ de altura. Raices fuertes, cespitosas, teretes, oscuras, de color verde oscuro, cubiertas de vainas. Seudobulbos carnosos, claviformes, que se van ensanchando gradualmente hacia el ápice redondeado, pendulosos, segmentados, recubiertos de vainas, $16-45 \mathrm{~cm}$ de largo por 3.5-6.0 $\mathrm{cm}$ de ancho. Hojas 4 por seudobulbo, con margen entero, nervaduras carinadas en el envés, de color verde brillante, plicadas, lanceoladas, agudas, con la base imbricada, $22-43 \mathrm{~cm}$ de largo por $5.6-9.4 \mathrm{~cm}$ de ancho. Inflorescencia en racimo que mide 37-45 $\mathrm{cm}$ de longitud, con 4-6 flores; el pedúnculo con brácteas, $3.4-4.5 \mathrm{~cm}$ de longitud. Flores de color amarillo, con manchas de color lila en el labelo. Sépalo dorsal arqueado, oblongo-elíptico, carnoso, 


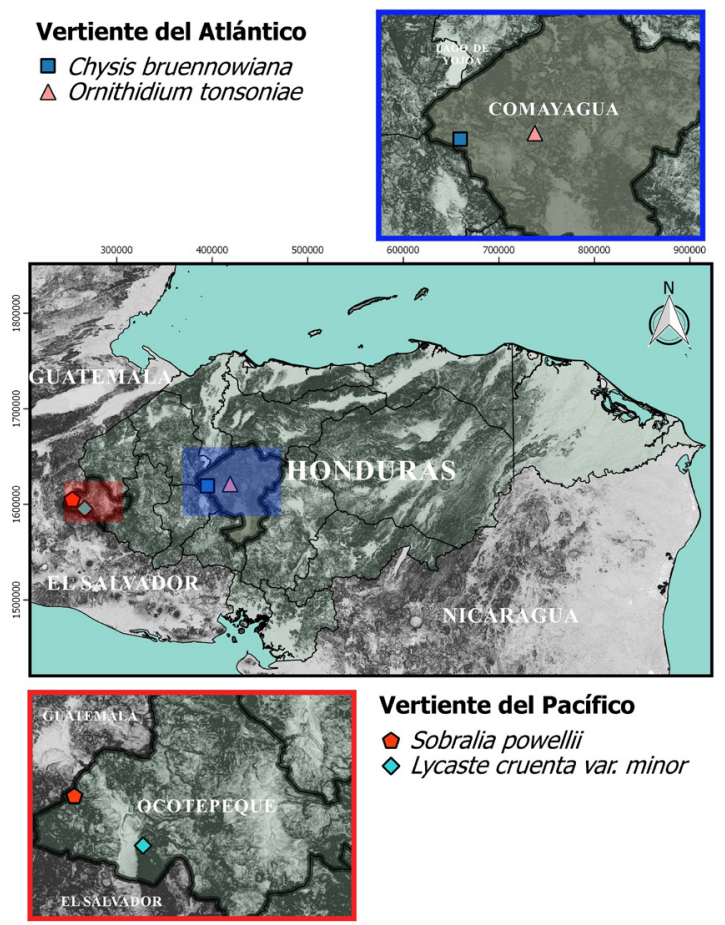

FIGURA 1. Ubicación de los nuevos registros en Honduras.

$3.0-3.5 \mathrm{~cm}$ de largo por $1.5-2.0 \mathrm{~cm}$ de ancho. Sépalos laterales oblongo-elípticos, unidos a la columna, connados en la base, formando un mentón, $2.0-2.5 \mathrm{~cm}$ de largo por $1.7-2.0 \mathrm{~cm}$ de ancho. Pétalos de obovados a lanceolados, $2.5-3.0 \mathrm{~cm}$ de largo por $1.0-1.5 \mathrm{~cm}$ de ancho. Labelo trilobado, reflexo, disco con tres quillas grandes y otra más pequeña a cada lado, todas de color blanco, carnosas; lóbulos laterales subfalcados, agudos de $2.0-2.5 \mathrm{~cm}$ de largo por $1.2-1.5 \mathrm{~cm}$ de ancho (Fig. 2A).

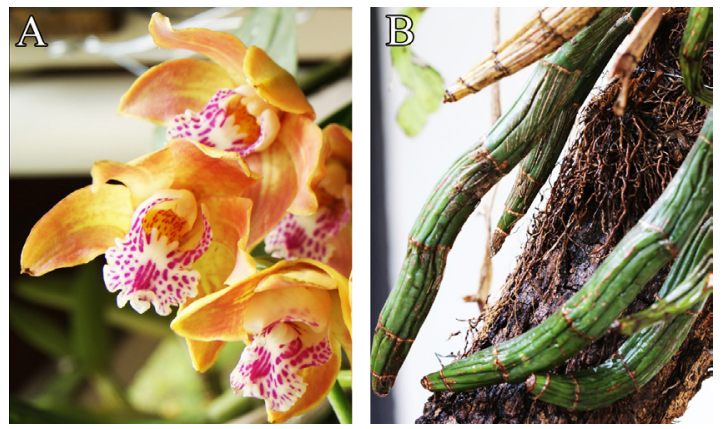

Figura 2. Características morfológicas de Chysis bruennowiana. A. Inflorescencia B. Pseudobulbos.
Reconocimiento: Planta grande y robusta, con seudobulbos claviformes, pendulosos, con el ápice redondeado. Labelo trilobado, reflexo, con cinco quillas carnosas de color blanco, las de los lados, más pequeñas. Las manchas color lila en el labelo son características de esta especie (Fig. 2A-B). Chysis tricostata Schltr., que se encuentra en forma simpátrica, es una planta más pequeña, con los seudobulbos de arqueados a erectos y posee manchas en el labelo de color rojo.

Adopto aquí el nombre de Chysis bruennowiana, el cual se ha usado en tratamientos recientes de las orquídeas del área mesoamericana (ej. Pupulin 2002, Dressler 3003, Ossenbach et al. 2007, Bogarín et al. 2014), aunque el tipo de esta especie es del Perú, una región que florísticamente comparte pocos elementos con la parte septentrional del istmo de Centro América.

Distribución: Honduras, Nicaragua, Costa Rica, Panamá, Perú y Bolivia.

Hábitat en Honduras: Hierba epífita de hasta $80 \mathrm{~cm}$ de altura, en árboles de bosque montano inferior y bosque tropical siempre verde (Mejía y House, 2002) (Fig. 6A) sobre árboles de café, en cafetal abandonado, junto a Chysis trichostata Schltr.

Fenología: Los tres especímenes estudiados florecieron en abril.

Material examinado: Honduras. Comayagua: cerca de la comunidad de El Rosario, Taulabé, 14³8'44.1'”N, 8758'34.1"O, $1153 \mathrm{~m}, 29$ de abril de 2016, D.E. Pérez 678 (EAP).

2. Lycaste cruenta var. minor Oakeley, Lycaste, Ida and Anguloa Essential Guide: 50-52. 2008. TIPO: Guatemala. En la colección de Otto Tinschert, ciudad de Guatemala, 4 de septiembre de 1991, Oakeley A63 (holotipo, K-HFO).

Hierba epipétrica, de hasta $23 \mathrm{~cm}$ de altura. Raices cespitosas, blanquecinas. Seudobulbos piriformes con vainas escariosas hasta la mitad del seudobulbo, 8-10 cm de largo por 6-7 $\mathrm{cm}$ de ancho. Flores color amarillo verdoso con labelo amarillo naranja, con fuerte esencia a vainilla. Inflorescencia $12-14 \mathrm{~cm}$ de longitud, con vainas triangulares. Sépalo dorsal cóncavo, acuminado, oblongo lanceolado, 3.5-3.8 $\mathrm{cm}$ de largo por 1.9-2.0 $\mathrm{cm}$ de ancho. Sépalos 

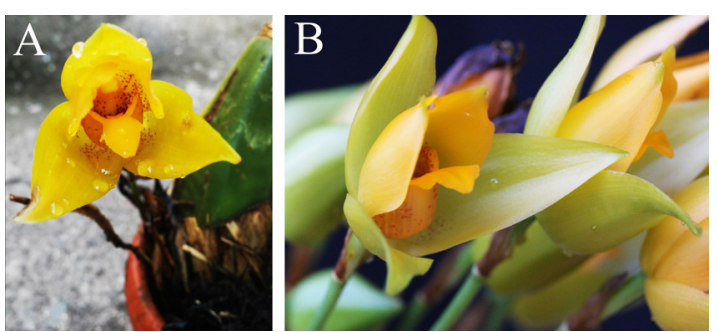

FiguRA 3. Características morfológicas de Lycaste cruenta var. minor. A. Inflorescencia. B. Flor.

laterales similares, $4-5 \mathrm{~cm}$ de largo por $1.5-2.3 \mathrm{~cm}$ de ancho. Pétalos superpuestos, ondulados, 1.5-3.0 $\mathrm{cm}$ de largo por $1.3 \mathrm{~cm}$ de ancho. Labelo trilobulado con la base con manchas de color rojo, la base del labelo del mismo largo que ancho, con un callo muy pequeño; el lóbulo central $1.0-1.2 \mathrm{~cm}$ de largo por $0.8-1.2 \mathrm{~cm}$ de ancho. Produce néctar, pero no tiene nectario.

ReConocimiento: el color rojo en la base del labelo, así como su tamaño pequeño (Fig. 3A-B), distingue a esta variedad. Difiere de Lycaste $\mathrm{x}$ cobani, en que esta última tiene la base del labelo más larga que ancha, los lóbulos laterales son elongados, lo que hace que los márgenes de enfrente se vean casi verticales (Oakeley 2008).

Distribución: Guatemala, El Salvador y Honduras. La especie ya estaba reportada para Honduras, no así la variedad, que fue determinada por H. Oakeley en 2016.

HÁBitat en Honduras: Hierba terrestre y litofítica, a lo largo de quebradas intermitentes, sobre rocas, en asociación con Sobralia sp. y Lycaste cochleata Lindl. Bosque montano inferior, de $1100 \mathrm{msnm}$, semicaducifolio (Mejía \& House 2002), con predominancia de tres tipos de robles (Fagaceae), Quercus segoviensis Liebm., Q. bumelioides Liebm., y $Q$. oleoides Schltdl. \& Cham. (Fig. 6B). Flores de color amarillo (Fig. 3A-B).

Fenología: Florece en enero, febrero, marzo y agosto.

Material examinado: Honduras. El Roblerón, municipio de Ocotepeque, Ocotepeque $14^{\circ} 25^{\prime} 15.3^{\prime} \mathrm{N}$, 8909'59.3"O, 1105 m, 3 de Marzo de 2016, D.E. Pérez et. al. 661 (EAP).
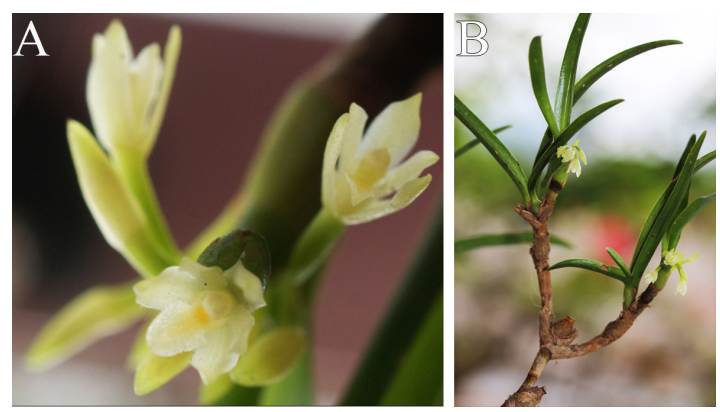

FIgURA 4. Características morfológicas de Ornithidium tonsoniae. A. Inflorescencia B. Seudobulbos.

3. Ornithidium tonsoniae (Soto Arenas) Senghas, Orchideen (ed. 3) I/C (44-45). 2001. TIPO: México. Chiapas: Mun. Ocosingo, $850 \mathrm{~m}, 20$ de julio de 1989, M.A.Soto Arenas, E.Martínez \& S.Purata 5710 (holotipo: AMO; isotipo: SEL).

Hierba epífita hasta $50 \mathrm{~cm}$ de alto. Raíces largas, teretes. Seudobulbos ovoides a globosos, de un solo entrenudo, progresivamente más pequeños hacia el ápice, $1.5-3.5 \mathrm{~cm}$ de largo por $1.0-2.5 \mathrm{~cm}$ de ancho. Tallos ramificados secundariamente, con vainas escariosas, imbricada, saliendo de la axila de las vainas foliáceas del seudobulbo. Inflorescencias 9-12, unifloras, producidas en grupos sobre los tallos; pedúnculo $1 \mathrm{~cm}$ de longitud. Flores de color blanco cremoso. Sépalo dorsal espatulado, $6 \mathrm{~mm}$ de largo por $3 \mathrm{~mm}$ de ancho. Sépalos laterales erectos, $10 \mathrm{~mm}$ de largo por $1 \mathrm{~mm}$ de ancho. Pétalos espatulados, $5 \mathrm{~mm}$ de largo por $2 \mathrm{~mm}$ de ancho. Labelo amarillo en la base, entero y triangular.

Reconocimiento: Soto Arenas (2002), reconoce esta especie por ser epífita sub arbustiva, con seudobulbos de ovoides a globosos, hojas carnosas, y las flores agrupadas y de color crema (Fig. 4A-B).

Distribución: México, Guatemala y Honduras.

HÁbitat en Honduras: Epífita de flores color blanco cremoso, (Fig. 4A) en bosque mesófilo de montaña. (Fig. 6C).

Fenología: Florece en diciembre.

Material examinado: Honduras. Comayagua: Montaña Los Imposibles, hacia Santa Rita de El Rosario,

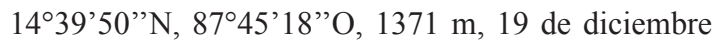
2015, D. E. Pérez, 651 (EAP).

LANKESTERIANA 17(3). 2017. (C) Universidad de Costa Rica, 2017. 

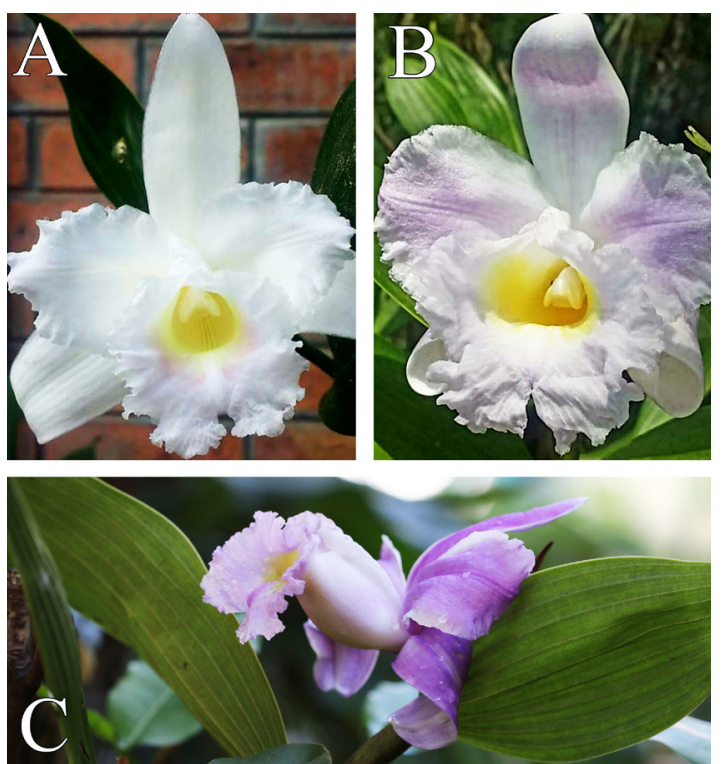

Figura 5. A, B y C: distintos colores y tonalidades de Sobralia powellii.
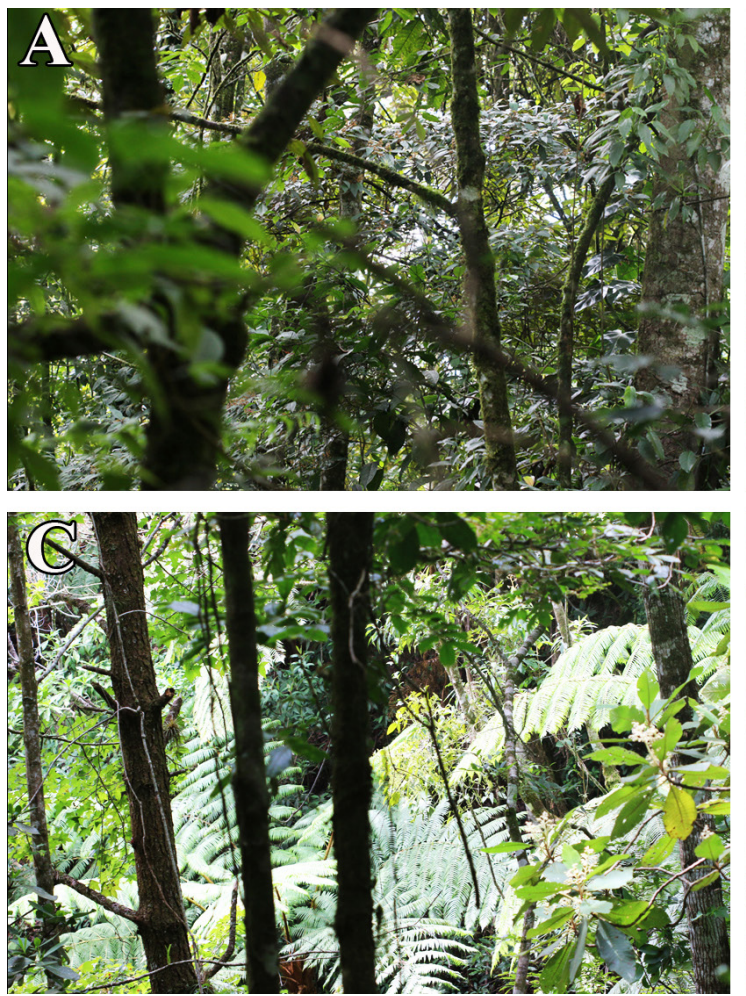

Figura 6. Hábitats donde crecen los nuevos registros de Honduras. A Chysis bruennowiana. B. Lycaste cruenta var. minor. C. Ornithidium tonsoniae. D. Sobralia powellii.

4. Sobralia powellii Schltr., Repert. Spec. Nov. Regni. Veg. Beih. 17: 11. 1922. TIPO: Panamá. An den hinterwässern des Gatun-Sees, C. W. Powell 2 (holotype, B, destroyed; lectotype designated by Christenson [1991], AMES, isolectotypes, MO).

Hierba terrestre, robusta, formando grandes masas, de $106-175 \mathrm{~cm}$ de altura. Raices gruesas, café. Tallos delgados, tipo caña, con vainas entre los entrenudos, escariosas y de color café. Hojas alternas, conduplicadas, de 4-10 por tallo, oblicuas, elípticas, fuertemente acuminadas, multinervadas (9-10), $13-22 \mathrm{~cm}$ de largo por $6-8 \mathrm{~cm}$ de ancho, de color verde claro con vainas foliares punteadas. Inflorescencia solitaria, con flores vistosas de color blanco cremoso, con bandas lila tanto en los sépalos como en los pétalos; garganta amarilla. Sépalo dorsal retuso, oblongo, acuminado, márgenes lisos, $6 \mathrm{~cm}$ de largo por $2 \mathrm{~cm}$ de ancho. Sépalos laterales de lanceolados a oblongo lanceolados, horizontales, retusos, 4-6 cm de largo por 2.0-2.5 cm de ancho. Pétalos anchos, oblongos a obovados, $4.5-5.0 \mathrm{~cm}$ de largo por $3 \mathrm{~cm}$ de ancho. Labelo doblado alrededor de la columna
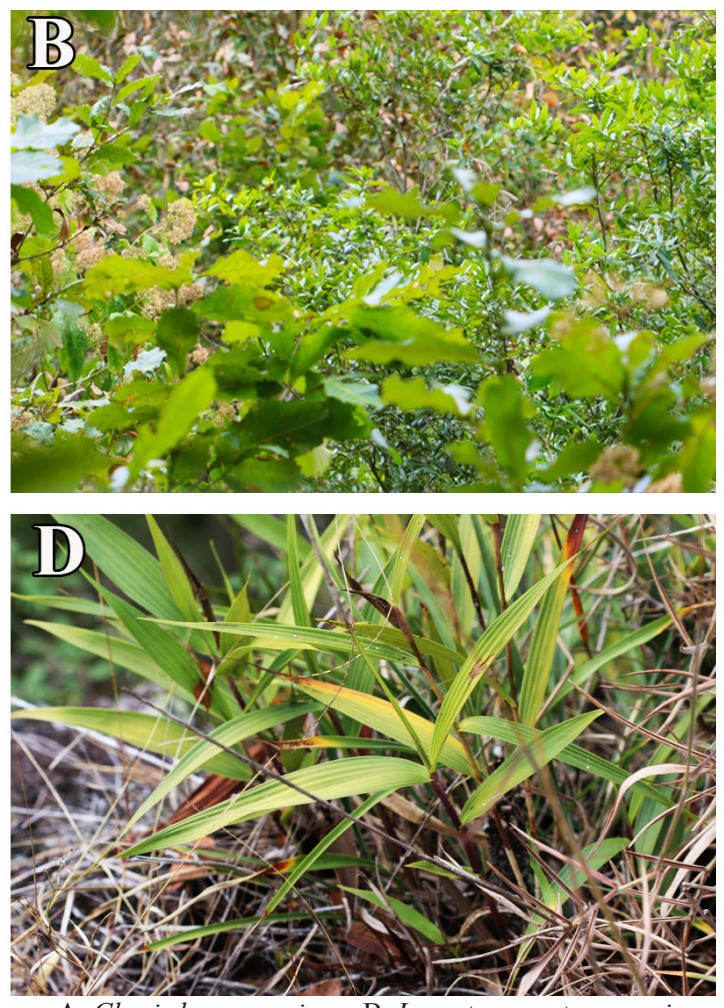

ANKESTERIANA 17(3). 2017. (C) Universidad de Costa Rica, 2017. 
como trompeta, o embudo, algo retuso, con márgenes sumamente ondulados (Fig. 5A-C).

RECONOCIMIENTO: Dodson (1984) reconoce esta especie por su tamaño grande, robusto, con flores grandes de color blanco, con el labelo amarillo y las líneas blancas en la porción expandida y reflexa de la porción apical del labelo (Fig. 5A-C). Por su tamaño puede confundirse con Sobralia macrantha Lindl., pero ésta tiene colores sólidos, sin bandas.

Distribución: Honduras, Nicaragua, Costa Rica, Panamá, Colombia, Ecuador y Perú.

HÁBITAT EN Honduras: Terrestre y rupícola, en cañones de quebradas y planicies cerca de las quebradas. Bosque tropical semicaducifolio, montano inferior, 1100-1200 msnm; bosque de pino (Pinaceae) y roble (Fagaceae) con Pinus oocarpa Schiede ex Schltdl. y Quercus oleoides, Q. sapotifolia Liebm., Byrsonima crassifolia (L.) Kunth (Malpighiaceae) (Mejía y House, 2002) (Figura 6D). Flores grandes con un conjunto de colores y tonalidades desde casi blanco, lila, hasta casi violeta (Figura 5A-C).

FENOLOGÍA: Florece en enero, febrero, y marzo.

Material eXaminado: Ocotepeque: Piedras Bonitas, $14^{\circ} 30^{\prime} 11.1^{\prime \prime} \mathrm{N}$ y $89^{\circ} 17^{\prime} 11.9^{\prime \prime O}, 1179 \mathrm{~m}, 31$ de enero 2016, D. E. Pérez et. al. 657 (EAP).

Agradecimientos. La autora agradece a Henry Oakeley por la identificación de Lycaste cruenta var. minor. A Franco Pupulin, por su ayuda en la identificación de especies y comentarios y sugerencias al manuscrito. A Alejandro Giggey, Diego Pavón y Leonardo Pérez Salles por la ayuda en la elaboración de mapas y figuras. En especial a Fray Jorge Aguilar Linares por su ayuda prestada en los viajes de campo, así como sus comentarios en cuanto a los sitios de colecta y revisión de los datos y las fotografía proporcionadas de Sobralia powellii. Al comité editorial por sus comentarios y sugerencias que enriquecieron el manuscrito.

\section{LITERATURA CITADA}

Bogarín, D., Z. Serracín, Z. Samudio, R. Rincón \& F. Pupulin (2014). An updated checklist of the orchidaceae of Panama. Lankesteriana, 14(1), 135-164.

Christenson, E. A. (1991). Mesoamerican orchid studies II: Powell's Panamian Orchidaceae. Lindleyana, 6(3), 124-132.

Dressler, R. L. (2003). Orchidaceae. Pp. 1-595 in: B. E. Hammel, M. H. Grayum, C. Herrera \& N. Zamora (eds.), Manual de Plantas de Costa Rica. Vol. 3. Monographs in Systematic Botany of Missouri Botanical Garden 93.

Dressler, R. L. (2011). ¿Las Sobralias se pueden clasificar? - El complejo de Sobralia warszewiczii. Lankesteriana, 11(3), 239-243.

Dodson, C.H. (1984). Icones Plantarum Tropicarum. The Marie Selby Botanical Gardens. Sarasota, FL.

Hágsater, E. \& M. A. Soto Arenas. (2002). Icones Orchidareum. 5-6, Plate 620. Mexico City.

Heywood, V. H. (1995). Global Biodiversity Assessment. United Nations Environment Programme. Cambridge University Press.

Linares, J. L. y P. Ancalmo (2016). Una nueva especie de Sobralia (Orchidaceae) de Honduras. Journal of the Botanical Research Institute of Texas, 10 (1). 1-7.

Nelson Sutherland, C. H. (2008). Catálogo de plantas vasculares de Honduras, espermatofitas. Editorial Guaymuras, Tegucigalpa.

Oakeley, H. F. (2008). Lycaste, Ida and Aguloa. The essential guide. Cambrian Printers. United Kingdom.

Ossenbach, C., F. Pupulin \& R. L. Dressler (2007). Orquídeas del Istmo Centroamericano. Catálogo y estado de conservación. Editorial 25 de mayo, Montes de Oca, San José, Costa Rica.

Pérez Munguía, D. E. (2015a). Orquídeas Nativas de Honduras, Vol. I. Editorial Ramsés, Tegucigalpa.

Pérez Munguía, D. E. (2015b). Orquídeas Nativas de Honduras, Vol. II. , Tegucigalpa.

Pupulin, F. (2002). Catálogo revisado y anotado de las Orchidaceae de Costa Rica. Lankesteriana, 4, 1-88

Vega, H., Cetzal-Ix, W., Mó, E., Germer, D. \& Soler, K. (2014). Nuevos Registros de Orchideaceae para Honduras. Lankesteriana, 14(2), 115-121.

Vega, H., Cetzal-Ix, W., Mó, E. \& Romero-Solera, K. (2016). Nuevos registros para la flora de Honduras y El Parque Nacional Montaña de Celaque. Acta biológica colombiana, 21(3), 635-644. 
LANKESTERIANA 